\title{
Expression of HMA4 cDNAs of the zinc hyperaccumulator Noccaea caerulescens from endogenous NcHMA4 promoters does not complement the zinc-deficiency phenotype of the Arabidopsis thaliana hma2hma4 double mutant
}

\author{
Mazhar Iqbal' *t, Ismat Nawaz' , Zeshan Hassan ${ }^{2}$, Henk W. J. Hakvoort ${ }^{1}$, Mattijs Bliek' ${ }^{1}$, Mark G. M. Aarts ${ }^{2}$ \\ and Henk Schat ${ }^{1}$
}

${ }^{1}$ Department of Genetics, Faculty of Earth and Life Sciences, Vrije Universiteit, Amsterdam, Netherlands

${ }^{2}$ Laboratory of Genetics, Wageningen University, Wageningen, Netherlands

Edited by:

Marc Hanikenne, University of Liège Belgium

\section{Reviewed by:}

Nathalie Verbruggen, Université Libre de Bruxelles, Belgium

Qingqing Xie, University of California, San Diego, USA

\section{*Correspondence:}

Mazhar lqbal, Department of

Genetics, Faculty of Earth and Life

Sciences, Vrije Universiteit, De

Boelelaan 1085, 1081 HV, Amsterdam,

Netherlands

e-mail:mazz366@gmail.com

${ }^{\dagger}$ Present address:

Mazhar lqbal, Department of Environmental Sciences, Faculty of Biological Sciences, Quaid-i-Azam University, Islamabad, Pakistan e-mail:mazz366@gmail.com
Noccaea caerulescens $(\mathrm{NC})$ exhibits a very high constitutive expression of the heavy metal transporting ATPase, HMA4, as compared to the non-hyperaccumulator Arabidopsis thaliana (At), due to copy number expansion and altered cis-regulation. We screened a BAC library for HMA4 and found that HMA4 is triplicated in the genome of a $N$. caerulescens accession from a former $\mathrm{Zn}$ mine near La Calamine (LC), Belgium. We amplified multiple HMA4 promoter sequences from three calamine $N$. caerulescens accessions, and expressed AtHMA4 and different NcHMA4 cDNAs under At and No HMA4 promoters in the A. thaliana (Col) hma2hma4 double mutant. Transgenic lines expressing HMA4 under the At promoter were always fully complemented for root-toshoot $\mathrm{Zn}$ translocation and developed normally at a $2-\mu \mathrm{M} Z \mathrm{n}$ supply, whereas the lines expressing HMA4 under Nc promoters usually showed only slightly enhanced root to shoot $\mathrm{Zn}$ translocation rates in comparison with the double mutant, probably owing to ectopic expression in the roots, respectively. When expression of the $\mathrm{Zn}$ deficiency responsive marker gene ZIP4 was tested, the transgenic lines expressing AtHMA4 under an NcHMA41-LC promoter showed on average a 7-fold higher expression in the leaves, in comparison with the double hma2hma4 mutant, showing that this construct aggravated, rather than alleviated the severity of foliar Zn deficiency in the mutant, possible owing to expression in the leaf mesophyll.

Keywords: Noccaea caerulescens, HMA4, promoter activity, Zn deficiency, Zn translocation, gene expression

\section{INTRODUCTION}

Zinc $(\mathrm{Zn})$ is an essential element for all organisms. However, it is toxic when taken up in excess (Marschner, 1995; Kamal et al., 2004). Therefore, all organisms tightly regulate their cellular $\mathrm{Zn}$ status (Clemens, 2001). The network underlying $\mathrm{Zn}$ homeostasis in plants is incompletely known, but a number of $\mathrm{Zn}$ transporters of the ZIP/IRT, MTP, and HMA families have been shown to play essential roles in the acquisition, plant-internal transport and sequestration of $\mathrm{Zn}$ (Guerinot, 2000; Hussain et al., 2004; Papoyan and Kochian, 2004; van de Mortel et al., 2006; Gustin et al., 2009; Wong and Cobbett, 2009; Shahzad et al., 2010). Heavy metal transporting ATPases (HMAs) constitute the $\mathrm{P}_{1 \mathrm{~b}}$ subfamily of cation transporter ATPases. In Arabidopsis thaliana this subfamily is represented by eight members, of which HMA1 - HMA4 and HMA5 - HMA8 are transporting divalent and univalent heavy metal cations, respectively. All HMAs are effluxing heavy metal ions from the cytosol, either into the apoplast, the vacuole, or other organelles (Hussain et al., 2004; Andrés-Colás et al., 2006; Kim et al., 2009; Ueno et al., 2011). In A. thaliana, HMA2 and HMA4 are plasma membrane-localized, particularly expressed in the xylem parenchyma of the roots, and supposed to be involved in the loading of $\mathrm{Zn}$ and Cd into the xylem (Hussain et al., 2004; Wong and Cobbett, 2009). They seem to be partly redundant, since the single knock-out mutants, hma4 and hma2, have only a modest or no phenotype for $\mathrm{Zn}$ root-to-shoot transport respectively, whereas the double mutant has a very strong phenotype under normal Zn supply, including stunted growth, chlorosis and infertility (Hussain et al., 2004).

A minority of plant species, called metallophytes, are capable to grow and reproduce on strongly heavy-metal enriched "metalliferous" soils. These plants, or at least their metallicolous populations, exhibit extraordinary high levels of tolerance, also called hypertolerance (Clemens, 2006), to particular heavy metals (Antonovics et al., 1971; Ernst, 1974; Macnair, 1993). In so-called facultative metallophytes or pseudometallophytes, i.e., species occurring on non-metalliferous as well metalliferous soils, hypertolerance is largely metal-specific and confined to the metal or metals present at toxic concentrations in the soil at the site of population origin (Schat et al., 1996; Schat and Vooijs, 1997). A small fraction of metallophytes, about 450 species worldwide, are classified as metal 
hyperaccumulator plants, accumulating particular heavy metals at extremely high concentrations in their foliage (Baker et al., 2000; van der Ent et al., 2013). Most of them hyperaccumulate nickel (Ni), but some of them mostly $\mathrm{Zn}$, and/or cadmium (Cd), such as Noccaea caerulescens (formerly known as Thlaspi caerulescens) and Arabidopsis halleri (Cosio et al., 2004).

The mechanisms of hypertolerance and hyperaccumulation in metallophytes are far from completely understood. However, $\mathrm{Cd}$ and $\mathrm{Zn}$ hyperaccumulation and hypertolerance in Arabidopsis halleri have been shown to depend on a strongly enhanced expression of HMA4, which is in this species effected by tandem triplication and altered cis-regulation in comparison to A. thaliana (Courbot et al., 2007; Willems et al., 2007; Hanikenne et al., 2008). Recently, enhanced HMA4 expression, due to tandem quadruplication and altered cis-regulation, has also been demonstrated in Noccaea caerulescens, which is a remarkable case of parallel molecular evolution, since the hyperaccumulation trait must have been independently evolved in Noccaea and Arabidopsis (Ó Lochlainn et al., 2011).

Although enhanced HMA4 expression is doubtlessly essential for the hypertolerance and foliar allocation of $\mathrm{Zn}$ and $\mathrm{Cd}$ in hyperaccumulators, as convincingly demonstrated by RNAi-mediated silencing in A. halleri (Hanikenne et al., 2008), it does not seem to be sufficient to confer significant levels of $\mathrm{Cd}$ or $\mathrm{Zn}$ hypertolerance or hyperaccumulator-like foliar accumulation rates in a non-hyperaccumulator/non-metallophyte genetic background. Heterologous expression of AhHMA4, under the AhHMA4 promoter, yielded enhanced $\mathrm{Zn}$ or $\mathrm{Cd}$ sensitivity, manifested as reduced shoot growth and chlorosis, but without considerably enhanced foliar metal accumulation in A. thaliana (Hanikenne et al., 2008) and tomato (Barabasz et al., 2012). The reason for this is still elusive, and further characterization of the functioning of hyperaccumulator HMA4 genes in a non-hyperaccumulator genetic background is therefore required. Moreover, although the three AhHMA4 copies seem to show very similar expression patterns (Hanikenne et al., 2008), it cannot be excluded that there is some degree of functional differentiation among them. Therefore, in the present study we made an attempt to more precisely characterize the HMA4 cDNAs and HMA4 promoters from Noccaea caerulescens, through expression in the A. thaliana hma2hma4 double mutant. We were particularly interested in the potential of NcHMA4 to revert the foliar $\mathrm{Zn}$ deficiency phenotype of the double mutant. We also phenotyped the transgenic lines for $\mathrm{Cd}$ tolerance and translocation. To better understand the function of HMA4 in hyperaccumulators and non-hyperaccumulators, we expressed the NcHMA4 cDNAs under the AtHMA4 promoter and AtHMA4 under NcHMA4 promoters, and compared the $\mathrm{Zn}$ and Cd translocation phenotypes of the transgenic lines with those of lines expressing AtHMA4 under the native AtHMA4 promoter. To detect potential differences in tissue or cell type specificity, we made the promoter::GUS constructs for the N. caerulescens HMA4 promoters and the $A$. thaliana one and compared their activities.

\section{MATERIALS AND METHODS}

\section{PLANT MATERIALS AND EXPERIMENTAL CONDITIONS}

Seeds of $A$. thaliana ( $\mathrm{Col}$ ) wild-type, the hma2hma4 double mutant and transgenic lines were sterilized in 96\% ethanol, then
$10 \%$ bleach, washed three times with sterilized water, suspended in $0.1 \%$ agarose and sown on $0.8 \%(\mathrm{w} / \mathrm{v})$ gelrite plates (Duchefa, G1101.0250) containing half-strength Murashige and Skoog (MS) medium at $\mathrm{pH} 5.7-5.9$ with $25 \mu \mathrm{g} / \mathrm{ml}$ hygromycin for the transgenic lines and no antibiotics for wild-type on square petri plates that were vertically placed. Seeds were germinated at $22^{\circ} \mathrm{C}$ under a $10 \mathrm{~h} \mathrm{day}^{-1}$ photoperiod. After 2 weeks seedlings were transferred to hydroponics culture in 1-L polyethylene pots (three plants per pot, each plant of a different genotype) containing a modified half-strength Hoagland's solution (Schat and Ten Bookum, 1992). Plants were grown in a climate room at $20 / 15^{\circ} \mathrm{C}$ day/night, light intensity $220 \mu \mathrm{mol} \mathrm{m}^{-2} \mathrm{~s}^{-1}$ at plant level, $10 \mathrm{~h} \mathrm{day}^{-1}, 75 \% \mathrm{RH}$. Nutrient solutions were renewed weekly. After 2 weeks in hydroponics, plants were exposed to five different concentrations of $\mathrm{Cd}$ $(0.5,12,25$ and $50 \mu \mathrm{M})$ and two concentrations of $\mathrm{Zn}$ (2 and $10 \mu \mathrm{M})$, supplied as $\mathrm{CdSO}_{4}$ or $\mathrm{ZnSO}_{4}$, ten plants per treatment. Before exposure, roots were stained with active carbon powder (to facilitate the measurement of root length increment) and washed with demineralised water (Schat and Ten Bookum, 1992). After five days of exposure, root growth, i.e., the length of the longest unstained root segment was measured.

Seeds of $N$. caerulescens, collected from the populations near La Calamine (LC), Belgium, and Saint Laurent de Miniers (this population is also known as Ganges, Ga) and Col du Mas de l'Aire (CMA), South-France, were sown on garden soil (Jongkind B.V., number 6, Aalsmeer, The Netherlands). Site and accession characteristics are given in Assunção et al. (2003; LC and Ga). CMA is another lead mine from the region around the village of $\mathrm{Ga}$. Some accumulation and tolerance characteristics of the accessions are given in Peer et al. (2003) and Mohtadi et al. (2012). Two-weeks-old seedlings were transferred to hydroponics in 1-L polyethylene pots containing a modified half-strength Hoagland's solution (Schat and Ten Bookum, 1992). After 2 weeks leaves and roots were harvested, snap-frozen in liquid nitrogen and stored at $-80^{\circ} \mathrm{C}$ until RNA extraction.

\section{DETERMINATION OF Cd AND Zn CONCENTRATIONS}

$\mathrm{Cd}$ and $\mathrm{Zn}$ concentrations were determined in roots and shoots (10 plants per population per concentration, pooled two by two, to make five samples) of wild-type, mutant and transgenic lines. Roots were carefully rinsed with ice-cold $\mathrm{PbNO}_{3}(5 \mathrm{mM})$ for $30 \mathrm{~min}$ and blotted with tissue paper. $\mathrm{Cd}$ and $\mathrm{Zn}$ were determined by digesting 50-100 mg of oven-dried plant material in $2 \mathrm{ml}$ of a 1:4 $(\mathrm{v} / \mathrm{v})$ mixture of $37 \%(\mathrm{v} / \mathrm{v}) \mathrm{HCl}$ and $65 \%(\mathrm{v} / \mathrm{v}) \mathrm{HNO}_{3}$ in Teflon bombs for $7 \mathrm{~h}$ at $140^{\circ} \mathrm{C}$, after which the volume was adjusted to $10 \mathrm{ml}$ with demineralised water. $\mathrm{Cd}$ and $\mathrm{Zn}$ were determined on a flame atomic absorption spectrophotometer (Perkin Elmer AAS100).

\section{RNA AND DNA EXTRACTION AND 1st STRAND cDNA SYNTHESIS}

RNA was extracted from frozen root and shoot tissues using Trizol $^{\mathrm{TM}}$ (Invitrogen), following the manufacturer's instructions and as described in Jack et al. (2007). Single-stranded cDNA was synthesized from total RNA $(2.5 \mu \mathrm{g}$, boiled for $1 \mathrm{~min})$ using 100 Units M-MLV Reverse Transcriptase (Invitrogen), $2 \mathrm{mM}$ dNTPs, $100 \mathrm{mM}$ DTT, $10 \times$ RT buffer and $10 \mu \mathrm{M}$ oligo dT primer at $42^{\circ} \mathrm{C}$ for $1 \mathrm{~h}$. DNA was isolated according to Rivera et al. (1999). 


\section{EXPRESSION ANALYSIS}

cDNA was synthesized following the manufacturer's protocol. Based on the sequence from the National Center for Biotechnology Information (NCBI) database ${ }^{1}$, intron spanning specific primers matching all of the HMA4 cDNAs were designed for real-time quantitative reverse transcriptase (RT) PCR (qRT-PCR; Table S1, Supplementary Material). ACT2 was used as a positive internal control. The position of the intron was predicted by aligning coding sequences of NcHMA4 with the AtHMA4. Quantitative RT-PCR was performed using SensiMix ${ }^{\mathrm{TM}}$ SYBR No-ROX kit (Bioline) using the Bio-Rad MJ Research Opticon ${ }^{\mathrm{TM}}$ Real Time PCR detection system (Life Technologies; Invitrogen). SensiMix ${ }^{\mathrm{TM}}$ SYBR No-ROX kit includes the SYBR ${ }^{\circledR}$ Green I dye, dNTPs, stabilizers and enhancers. A dilution range in water of the cDNA samples was tested to identify the cDNA concentration that produced a $\mathrm{C}_{\mathrm{T}}$ between 15 and 30 cycles. The final reaction conditions were, $10 \mu \mathrm{l}$ SensiMix ${ }^{\mathrm{TM}}$ SYBR No-ROX master mix, $0.75 \mu \mathrm{l}$ forward primer (final concentration of $250 \mathrm{nM}$ ), $0.75 \mu$ l of reverse primer (final concentration of $250 \mathrm{nM}$ ) and cDNA in a total reaction volume of $20 \mu \mathrm{l}$. An initial step of $95^{\circ} \mathrm{C}$ for $10 \mathrm{~min}$ was used to activate the polymerase. Cycling conditions were: melting step at $95^{\circ} \mathrm{C}$ for $10 \mathrm{~s}$ and annealing-extension at $60^{\circ} \mathrm{C}$ for $20 \mathrm{~s}$, with 40 cycles, at the end melting curve from 60 to $90^{\circ} \mathrm{C}$, read every $0.5^{\circ} \mathrm{C}$, hold $10 \mathrm{~s}$. All qPCR reactions were performed in triplicate, and a maximum difference of one cycle between the $\mathrm{C}_{\mathrm{T}}$ of the triplicate samples was considered acceptable. Negative controls were included for each primer pair to check for significant levels of any contaminants. Expression values were calculated using the $2^{-\Delta \Delta \mathrm{CT}}$ method (Livak and Schmittgen, 2001).

\section{DNA BLOT HYBRIDISATION}

We screened a BAC library of LC for HMA4. BAC clone DNA was extracted from $5 \mathrm{ml}$ of overnight culture grown in LB medium containing $12.5 \mu \mathrm{g} / \mathrm{ml}$ chloramphenicol, using the Miniprep Plasmid DNA Purification kit (QIAGEN) but skipping the column purification step. For Southern analyses, $2 \mu \mathrm{g}$ of BAC clone DNA from two clones was digested with restriction enzymes (EcoRV, XbaI, BamHI, HindIII, EcoRI and PstI) at $37^{\circ} \mathrm{C}$ for $6-$ $7 \mathrm{~h}$ and separated on a $0.8 \%(\mathrm{w} / \mathrm{v})$ agarose gel in full-strength TEA buffer (20 mM Tris-acetate; $1 \mathrm{mM}$ EDTA). Then, the agarose gel was submerged into $0.25 \mathrm{~N} \mathrm{HCl}$ for $15 \mathrm{~min}$ and rinsed with water 2-3 times for $15 \mathrm{~min}$. DNA fragments were transferred and cross-linked onto a positively charged nylon membrane (Hybond-N+, Amersham Biosciences) by capillary blotting using $0.4 \mathrm{~N} \mathrm{NaOH}$ overnight $( \pm 16 \mathrm{~h})$. For DNA blot analysis of BAC clones, an NcHMA4 probe was obtained from a PCR fragment of $521 \mathrm{bp}$ produced from LC genomic DNA using forward (5'-ACAGGAAGAAAGTTGAAGGCGG-3') and reverse (5'-CCTCACTAGCAA-GCAACAAACG-3') primers designed on the last exon and purified using PCR purification kit (QIAGEN). Fifty ng of purified PCR product was radioactively labeled with $\alpha\left[{ }^{32} \mathrm{P}\right] \mathrm{dCTP}$ using the Klenow fragment of DNA polymerase with random hexamer primers. Prehybridization was carried out in Gilbert and Clark buffer (0.5 M phosphate buffer, $\mathrm{pH} 7.2 ; 7 \%$ SDS; $1 \% \mathrm{BSA}$ ) for $2 \mathrm{~h}$. Hybridization was carried out in the same buffer overnight at $65^{\circ} \mathrm{C}$. Then the blot was washed at $55^{\circ} \mathrm{C}$ with $2 \times$ SSC and $0.1 \%$ SDS. The final and more stringent wash was
$0.5 \times$ SSC, $0.1 \%(\mathrm{w} / \mathrm{v}) \mathrm{SDS}$, again at $55^{\circ} \mathrm{C}$ (Sambrook et al., 1989). Images were made using a Storm 820 Phospho-imager.

\section{GENERATION AND CHARACTERIZATION OF TRANSFORMED LINES}

Several chromosome walks were performed using DNA of $\mathrm{N}$. caerulescens, accessions LC, Ga and CMA, to pick up the promoters of HMA4 using primers listed in Table S2 (Supplementary Material). NcHMA4-Ga promoter 1 and 2 were amplified using forward primers designed on the promoter sequence of Saint Laurent Le Minier HMA4 (Ó Lochlainn et al., 2011). AtHMA4 cDNA was amplified from a cDNA clone obtained from Riken, Japan (Resource number pda 08214, cDNA clone RAFL 09-32D05). The sequence from the start codon of AtHMA4 until the upstream gene was taken as the AtHMA4 promoter (82748808278980 and 3 nucleotides of $5^{\prime}$ UTR just before ATG in TAIR ${ }^{2}$. Amplicons with the Noccaea promoters fused with the AtHMA4 and NcHMA4 cDNAs, the AtHMA4 promoter with the NcHMA4 and AtHMA4 cDNAs, and HMA4 promoters were prepared using attB1 and attB2 sites flanking the forward and reverse primers. In case of promoter::cDNA fused products, promoters and cDNAs were amplified separately with the overlapping parts at the fusion side and then fused in a single PCR (10 cycles without primers [as the overlapping parts will elongate to complete the fragment], then 25 cycles with atB1 forward and attB2 reverse primer to amplify the whole fragment). PCR reactions were performed using the "Phusion ${ }^{\circledR}$ High Fidelity DNA Polymerase" (Finnzymes). The primers used to amplify these products are given in the Supplementary Material under "Supplementary methods." All DNA recombinant techniques were performed according to the Invitrogen GATEWAY Cloning System (Karimi et al., 2002). We used pHGWFS7 as a destination vector for promoter analysis, and pH7WG2 without p35S for all other constructs (Karimi et al., 2002). All the fragments were confirmed by sequencing. Transformation with these binary vectors confers resistance to hygromycin in transformed plant cells. These binary vectors were introduced into the Agrobacterium tumefaciens strain C58 (pMP90) by electroporation.

Seeds of the homozygous A. thaliana hma2hma4 double mutant (Col; Hussain et al., 2004) were kindly provided by Prof. Chris Cobbett, University of Melbourne. A.thaliana (wt) and Athma2hma4 double mutants were transformed by A. tumefaciens containing the promoter and all other constructs, respectively, given in Table 1, using the flower dip technique (Clough and Bent, 1998). $\mathrm{T}_{0}$ seeds (three lanes with \pm 1000 seeds each) were surface-sterilized and sown on $0.8 \%(\mathrm{w} / \mathrm{v})$ gelrite plates containing half-strength MS medium at $\mathrm{pH}$ 5.7-5.9 with $50 \mu \mathrm{g} / \mathrm{ml}$ hygromycin for screening. The plates were kept vertically to record the root growth. After 2 weeks, there was a clear difference in root growth between the transgenic and untransformed plants. The transgenic plants were transferred to a nutrient solution containing a modified half-strength Hoagland's nutrient solution (see above). After 2 weeks in hydroponics, samples were taken from roots and leaves to extract RNA. RNA was isolated using Trizol. cDNA was synthesized using M-MLV from Invitrogen. Then the relative transcript levels were measured by RT-qPCR, using actin-2 as a positive internal control (see above). The primers are given in Table S1, Supplementary Material. 
Table 1 | Constructs used for $\boldsymbol{A}$. thaliana transformation and phenotyping experiments. For HMA4, cDNA was used.

\begin{tabular}{|c|c|}
\hline pAtHMA4::GUS & pAtHMA4::NcHMA4-2-LC \\
\hline pNcHMA4-1-LC::GUS & pNcHMA4-1-LC::NcHMA4-1-LC \\
\hline pNcHMA4-2-LC::GUS & pNcHMA4-1-LC::NcHMA4-2-LC \\
\hline pNcHMA4-3-LC::GUS & pNcHMA4-1-LC::AtHMA4 \\
\hline pNcHMA4-1-Ga::GUS & pNcHMA4-2-LC::AtHMA4 \\
\hline pNcHMA4-2-Ga::GUS & pNcHMA4-3-LC::AtHMA4 \\
\hline pNcHMA4-3-Ga::GUS & pNcHMA4-1-Ga::AtHMA4 \\
\hline pNcHMA4-1-CMA::GUS & pNcHMA4-2-Ga::AtHMA4 \\
\hline pNcHMA4-2-CMA::GUS & pNcHMA4-3-Ga::AtHMA4 \\
\hline pAtHMA4::AtHMA4 & pNcHMA4-1-CMA::AtHMA4 \\
\hline pAtHMA4::NCHMA4-1-LC & pNcHMA4-2-CMA::AtHMA4 \\
\hline
\end{tabular}

pNcHMA4-1-LC and $p N c H M A 4-2-L C$ were used in a LR reaction with the pKGWGG-RR vector (Limpens etal., 2004) through Gateway ${ }^{\circledR}$ LR Clonase ${ }^{\mathrm{TM}}$ II Enzyme Mix (Invitrogen ${ }^{\mathrm{TM}}$ ) to create an expression clone. Sequence reactions were done using the big dye terminator protocol. These expression clones were inserted separately in Agrobacterium rhizogenes by electroporation, and then used for hairy root transformation of $N$. caerulescens.

\section{HAIRY ROOT TRANSFORMATIONS OF $\boldsymbol{N}$. CAERULESCENS}

Roots of $N$. caerulescens were transformed with pNcHMA4-LC constructs via Agrobacterium rhizogenes mediated transformation. Single colonies of transformed A. rhizogenes (MSU 440) were cultured in $3 \mathrm{ml} \mathrm{LB}$ media with $100 \mu \mathrm{g} / \mathrm{ml}$ spectinomycin at $28^{\circ} \mathrm{C}$ overnight. Two hundred $\mu \mathrm{l}$ from the liquid culture were plated on LB-agar plates containing the same antibiotic and grew for 2 days at $28^{\circ} \mathrm{C}$. Seeds of $N$. caerulescens accession LC were sown in four agar plates containing half-strength MS ( $\mathrm{pH}$ 5.8), 25 seeds per plate. Seven-days-old plants had their roots removed just below the hypocotyl. One dot of aggregated $A$. rhizogenes bacteria was put at the tip of each hypocotyl. For co-culturing, plates were incubated in a climate chamber, set at $250 \mu \mathrm{mol} \mathrm{m} \mathrm{m}^{-2} \mathrm{~s}^{-1}$ light at plant level, during a 16$\mathrm{h}$ day period, $24^{\circ} \mathrm{C}$, and $70 \%$ relative humidity. After 5 days, seedlings were transferred to new $1 / 2$ MS-agar plates supplemented with $100 \mu \mathrm{g} / \mathrm{ml}$ tricarcillin to kill the bacteria. Roots were checked every three days using a stereomicroscope under UV, for expression of the DsRED protein indicating transformation. Nontransformed roots were removed. After 8 weeks, the plants showing fully transformed root systems were analyzed for GUS reporter activity.

\section{GUS ASSAY}

Seeds of $A$. thaliana primary transformants containing AtHMA4 or NcHMA4 promoter - GUS fusions were sown on $0.8 \%(\mathrm{w} / \mathrm{v})$ gelrite plates containing half-strength MS medium at $\mathrm{pH}$ 5.7-5.9 with $25 \mathrm{mg} \mathrm{l}^{-1}$ hygromycin. GUS activity was determined in 2week-old $A$. thaliana seedlings growing on MS gelrite plates, and in transformed $N$. caerulescens roots by staining overnight in a solution containing $1 \mathrm{mM}$ X-Gluc in $50 \mathrm{mM}$ sodium phosphate at $\mathrm{pH}$ 7.5. After the incubation, 70\% ethanol was used to remove chlorophyll. Staining patterns were observed and photographed using a binocular microscope (bright light).

\section{SEQUENCE AND PHYLOGENETIC ANALYSES}

Complete coding sequences of HMA4 were amplified using primers designed on the basis of the sequence available in the database (Bernard etal., 2004). Data base searches were conducted with BLAST ${ }^{1}$ and TAIR $^{2}$. The nucleotide sequences were aligned using ClustalW2 ${ }^{3}$. A phylogenetic tree was constructed using MEGA4 (Kumar et al., 2008) with Neighbor-joining and 1000 bootstrap replicates.

\section{STATISTICS}

Statistical analysis was performed using one-way and two-way ANOVA. The MSR statistic was used for a posteriori comparisons of individual means (Sokal and Rohlf, 1981). To obtain homogeneity of variances, all the data were subjected to logarithmic transformation prior to analysis, except for the root elongation data.

\section{RESULTS}

\section{DNA BLOT ANALYSIS}

A genomic BAC library of $N$. Caerulescens, LC, with an average insert size of $135 \mathrm{~Kb}$ was constructed by EPICENTRE Biotechnologies. Eight 384 well plates were screened for HMA4. We found two clones containing HMA4. DNA blot analysis showed that most of the lanes contained three bands, suggesting that HMA4 is at least triplicated in LC (Figure 1), because we used restriction enzymes that do not cut within the probed exon.

\section{N. CAERULESCENS HMA4 CODING AND PROMOTER SEQUENCES}

We constructed phylogenetic trees for NcHMA4s and other known HMA4 coding sequences and promoters (Figures S1 and S2, Supplementary Material). A. halleri HMA4 cDNAs are about $88 \%$ identical with the A. thaliana HMA4 cDNA (Hanikenne et al., 2008). N. caerulescens HMA4 cDNAs are less similar to the AtHMA4 cDNAs, sharing 80 to $85 \%$ identity. All the NcHMA4s share about $98 \%$ identity. On an amino acid basis AhHMA4s and NcHMA4s are around $86 \%$, or 71 to $76 \%$ identical with AtHMA4, respectively and NcHMA4s are 94 to 99\% identical among each other (Table S3-A, Supplementary Material).

When comparing promoter sequences, the NcHMA4 promoters are 36 to $39 \%$ identical with the AtHMA4 promoter, and 38 to $43 \%$ identical with the AhHMA4 promoters. When compared among each other, the NcHMA4 promoters share 36 to 96\% identity (Table S3-B and Alignment S3, Supplementary Material).

\section{EXPRESSION OF HMA4 IN A. THALIANA AND N. CAERULESCENS}

Transcript levels of HMA4 were determined by qRT-PCR in $A$. thaliana and $N$. caerulescens shoots and roots. In $A$.

\footnotetext{
${ }^{1}$ http://www.ncbi.nlm.nih.gov

${ }^{2} \mathrm{http}$ ///www.arabidopsis.org

${ }^{3}$ http://www.ebi.ac.uk/Tools/msa/clustalw2/
} 


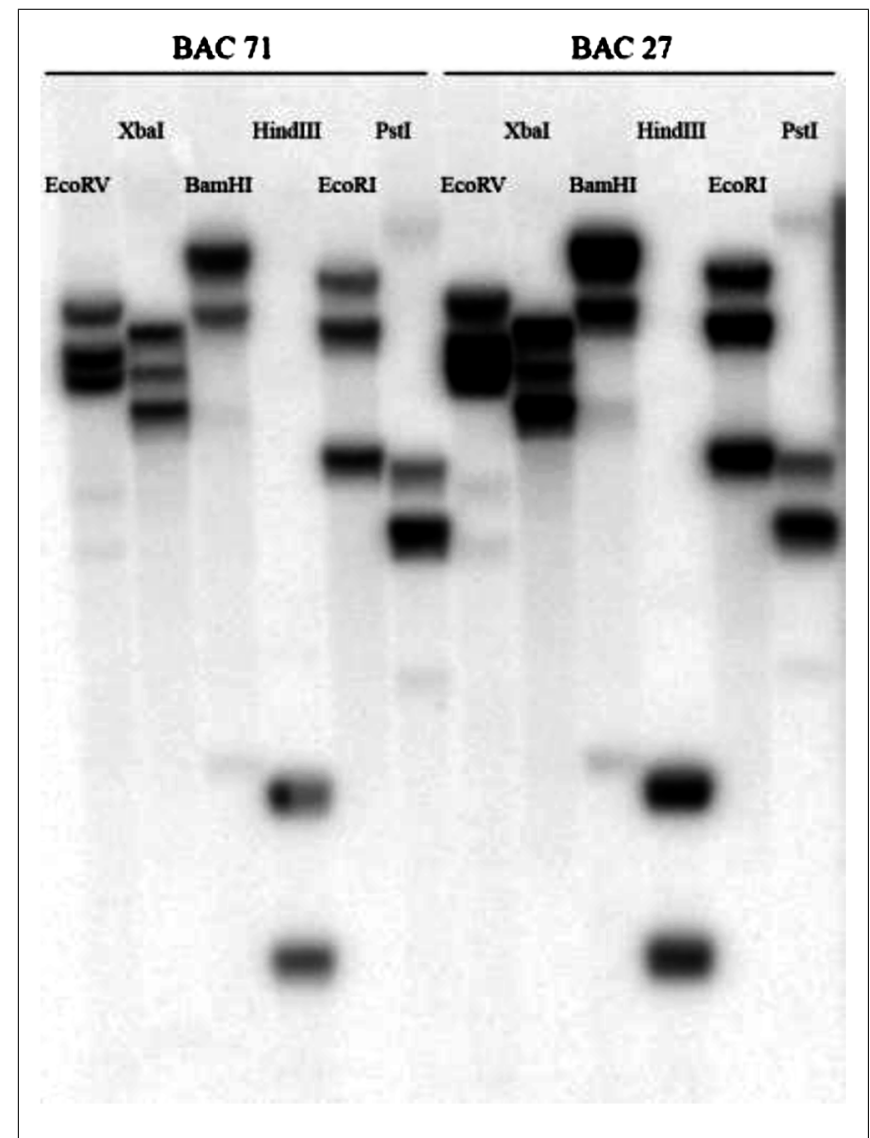

FIGURE 1 | Southern blot of two BAC clones of $\boldsymbol{N}$. caerulescens, accession La Calamine. Each clone was restricted by six different restriction enzymes (EcoRV, Xbal, BamHI, HindIII, EcoRI and Pstl). Hybridization was done with a 521 bp probe from the last exon of HMA4.

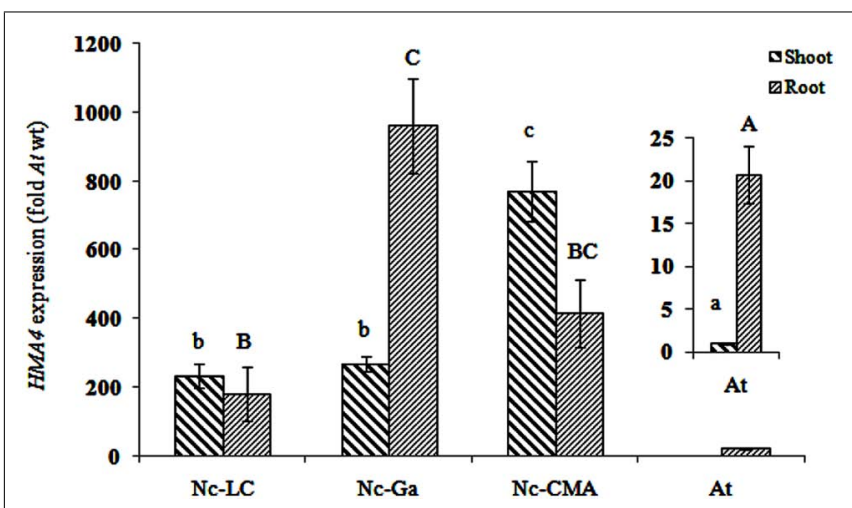

FIGURE 2 | HMA4 expression in three Noccaea caerulescens accessions (fold $A$. thaliana in shoot) La Calamine (LC), Ganges (Ga) and Col du Mas de I'Aire (CMA). Significant differences $(P<0.05)$ between means are indicated, separately for root and shoot, by different superscripted letters.

thaliana HMA4 was mainly expressed in the root, i.e., 20 times higher than in the shoot. In $N$. caerulescens LC and CMA HMA4 was higher expressed in the shoot than in the root, whereas in $\mathrm{Ga}$ it was higher expressed in the root
(Figure 2). Assuming equal expression of Act2, the shoot and root HMA4 transcript concentrations were about 400-fold and 25 -fold higher, respectively, in $N$. caerulescens than they were in A. thaliana.

\section{TRANSGENIC HMA4 EXPRESSION USING N. CAERULESCENS AND A. THALIANA HMA4 PROMOTERS}

We expressed NcHMA4-1-LC and AtHMA4 cDNAs under different NcHMA4 promoters and the AtHMA4 promoter in the Athma2hma4 double mutant. There was no significant effect of the cDNA source on the expression levels in 4-weeks old primary transgenic plants (Figures 3 and 4). When expressed under Nc promoters, the average transgene expression levels in the roots were, surprisingly, lower than those under the endogenous AtHMA4 promoter, except for the pNcHMA4-1-LC. The latter and the At promoter both yielded expression levels close to the wild-type level in untransformed A. thaliana (Figure 3). In the shoot, however, all the $N c$ promoters tested were significantly (4- to 60-fold) more active than the At one (Figure 4). To compare the patterns of tissue-specificity, we also expressed, under the same promoters, GUS in wild-type A. thaliana. When expressed under the AtHMA4 promoter, GUS activity was consistently high in the root stele, but negligible in the root tip. When expressed under NcHMA4 promoters, however, GUS activity was often low or negligible in the root stele, but (extremely) high all over the apical 2-mm root segment (Figure 5). Moreover, the NcHMA4 promoters often produced considerable GUS activity in the leaves, occasionally all over the leaf blade, rather than confined to the veins, whereas the AtHMA4 promoter was barely active in the leaves (Figure 5).

GUS was also expressed under the pNcHMA4-1-LC and pNcHMA4-2-LC in N. caerulescens, using A. rhizogenes-mediated root transformation. Staining was observed mainly in the stele, like $p A t H M A 4$, and the root tip, though exclusively in the root cap (Figure 6).

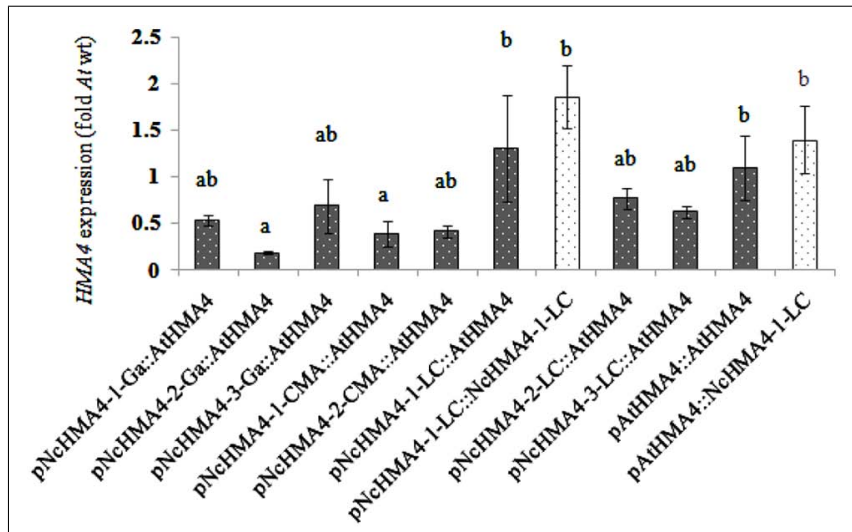

FIGURE 3 | AtHMA4 (solid bars) and NcHMA4-1-LC (open bars) expression under $A$. thaliana and $N$. caerulescens promoters in the root of transgenic $A$. thaliana hma2hma4 double mutants, relative to the average expression in the root of $A$. thaliana wild-type which is considered as 1 (means of 5 plants \pm SE). Significant differences $(P<0.05)$ between means are indicated by different superscripted letters. 


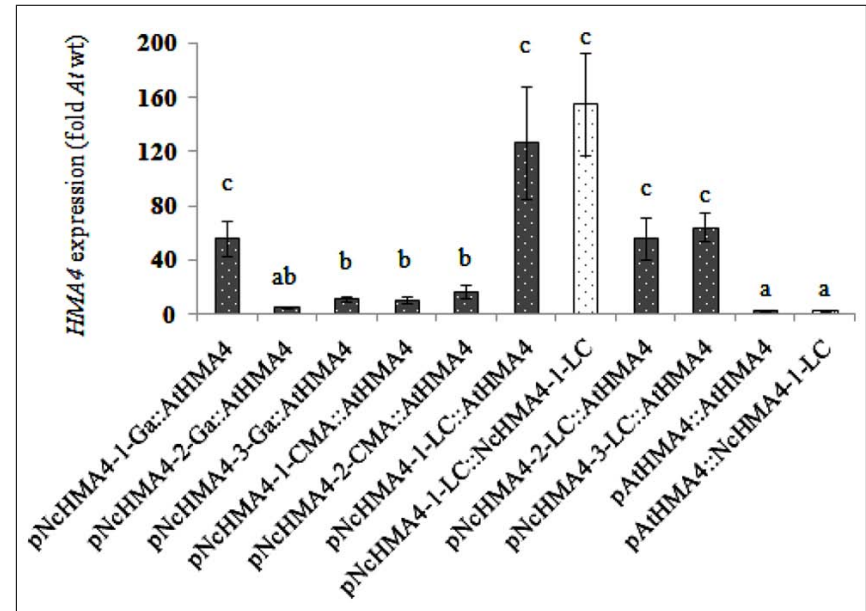

FIGURE 4 | AtHMA4 (solid bars) and NcHMA4-1-LC (open bars) expression under $A$. thaliana and $N$. caerulescens promoters in the shoot of transgenic $A$. thaliana relative to the expression in the shoot of $A$. thaliana wild-type which is considered as $\mathbf{1}$ (means of 5 plants \pm SE). Significant differences $(P<0.05)$ between means are indicated by different superscripted letters.

\section{MUTANT COMPLEMENTATION WITH Nc AND At HMA4 PROMOTER-DRIVEN HMA4 cDNAs}

All primary transgenic hma2hma4 double mutant plants expressing NcHMA4 or AtHMA4 cDNAs under the AtHMA4 promoter developed normally in a control nutrient solution ( $2 \mu \mathrm{M} \mathrm{Zn})$. On the other hand, with few exceptions, the majority of the $\mathrm{T}_{0}$ plants expressing NcHMA4 or AtHMA4 under any of the NcHMA4 promoters developed the stunted, curly and chlorotic rosette leaves typical of the Athma2hma4 double mutant. These plants were all infertile. In many of them the inflorescences already died before anthesis, which was also observed in case of the double mutant, and in the rest, the siliques remained very small $(<4 \mathrm{~mm})$ and completely devoid of seeds. Some plants developed more or less normal leaves and normally sized inflorescences, but with small and seedless siliques. All of these plants, including the untransformed double mutants, were rescued by increasing the $\mathrm{Zn}$ concentration in the nutrient solution from 2 to $10 \mu \mathrm{M}$. Only two transgenic plants, both transformed with the pNcHMA4-1-LC::AtHMA4 construct, developed normally and set seeds at $2 \mu \mathrm{M} \mathrm{Zn}$. To compare expression levels and phenotypes, we classified all pNcHMA4-1-LC::AtHMA4 plants, according to their $\mathrm{Zn}$ deficiency phenotype, into three categories, i.e., (1) no apparent $\mathrm{Zn}$ deficiency symptoms at $2 \mu \mathrm{M} \mathrm{Zn}$ ("normal"),

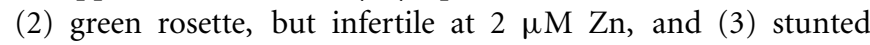
and chlorotic rosette and infertile at $2 \mu \mathrm{M} \mathrm{Zn} \mathrm{(Figures} \mathrm{7A,B).}$ The severity of the $\mathrm{Zn}$ deficiency phenotype appeared to be inversely related to the level of HMA4 expression in the root. The "normal" plants had expression levels of less than half of those of plants expressing AtHMA4 under the At promoter, on average. The green, but infertile, and the chlorotic infertile plants had expression levels close to, and higher than the mean for $p A t H M A 4:: A t H M A 4$-transformed plants, respectively (Figure 7A).

\section{ZN ROOT-TO-SHOOT TRANSLOCATION AND MUTANT COMPLEMENTATION}

We compared $\mathrm{Zn}$ root-to-shoot translocation among wildtype and hma2hma4 double mutant and transgenic plants. In a first experiment we used selected $T_{1}$ transgenic plants (10 plants per concentration) derived from $\mathrm{T}_{0}$ plants expressing AtHMA4 approximately at wild-type levels in the roots, under the pNcHMA4-1-LC, pNcHMA4-2-LC, pNcHMA4-3-Ga and $p N c H M A 4-1-C M A$, respectively. After 3 weeks of growth in hydroponics at $2 \mu \mathrm{M} \mathrm{Zn}$, all of the transgenic lines had foliar $\mathrm{Zn}$ concentrations that were slightly, but significantly higher than that of the double mutant, but much lower than that of wildtype plants (Figure 8A). The same pattern was observed at $10 \mu \mathrm{M}$ $\mathrm{Zn}$ (data not shown). The root $\mathrm{Zn}$ concentrations were highest in the double mutant and lowest in the wild-type, except for the pNcHMA4-1-CMA::AtHMA4 line at $10 \mu \mathrm{M} \mathrm{Zn}$ (data not shown). All the transgenic lines and the double mutant showed symptoms of foliar Zn deficiency.

In a second experiment we compared selected $\mathrm{T}_{1}$ derived from $\mathrm{T}_{0}$ plants transformed with pAtHMA4::AtHMA4, pAtHMA4::NcHMA4-1-LC, pAtHMA4::NcHMA4-2-LC, pNcHM A4-1-LC::NcHMA4-1-LC, pNcHMA4-1-LC::NcHMA4-2-LC, and pNcHMA4-1-LC::AtHMA4, using the wild-type as a reference. For this experiment we chose the $\mathrm{T}_{1}$ progenies derived from $\mathrm{T}_{0}$ plants expressing NcHMA4-LC 5- to 10-fold higher than wild-type in the roots. The two AtHMA4 expressing lines were derived from $\mathrm{T}_{0}$ plants with approximately wild-type expression levels in their roots. The lines with the constructs containing the AtHMA4 promoter had wild-type foliar $\mathrm{Zn}$ concentrations, or even higher in the case of the pAtHMA4::NcHMA4-2-LC line, which was derived from a $\mathrm{T}_{0}$ plant with a particularly high root expression level (six times At wild-type level, in comparison with 1.5 and 4 times for the $\mathrm{T}_{0}$ parents of the other lines; Figure $8 \mathbf{B}$ ). The lines with the constructs containing the pNcHMA4-1-LC exhibited foliar $\mathrm{Zn}$ concentrations that were significantly lower than $A t$ wild-type level. The two lines derived from the $\mathrm{T}_{0}$ plants with the highest root expression levels ( 9 and 10 times At wild-type level for $p N c H M A 4-$ 1-LC::NcHMA4-1-LC, pNcHMA4-1-LC::NcHMA4-2-LC) showed much higher foliar $\mathrm{Zn}$ concentrations, i.e., more than half of the wild-type level, than the one derived from the $\mathrm{T}_{0}$ with the lowest root expression level (1.5 times for pNcHMA4-1-LC::AtHMA4), i.e., about $25 \%$ of wild-type, comparable to the former experiment (Figure 8A). As expected, in the pNcHMA4-1-LC::AtHMA4 line the root $\mathrm{Zn}$ concentration was higher than in $A t$ wild-type, but this was also the case for the pAtHMA4::NcHMA4-2-LC line (Figure 8B). All the lines transformed with the pNcHMA4-1-LC, as well as the double mutant, showed symptoms of foliar Zn deficiency, whereas all the lines transformed with the At promoter did not.

To estimate the degree of foliar $\mathrm{Zn}$ deficiency in the lines transformed with the $p N c H M A 4-1-L C:: H M A 4$ construct, we measured the foliar expression level of a $\mathrm{Zn}$ deficiency sensitive marker gene, ZIP4 (Assunção et al., 2010), in an pNcHMA4-1-LC::AtHMA4 $\mathrm{T}_{1}$ line, the hma2hma4 double mutant, and the wild-type. ZIP4 was five fold higher expressed in the double mutant, and even 35fold higher in the transgenic line, in comparison with wild-type, showing that expression of HMA4 under the pNcHMA4-1-LC 


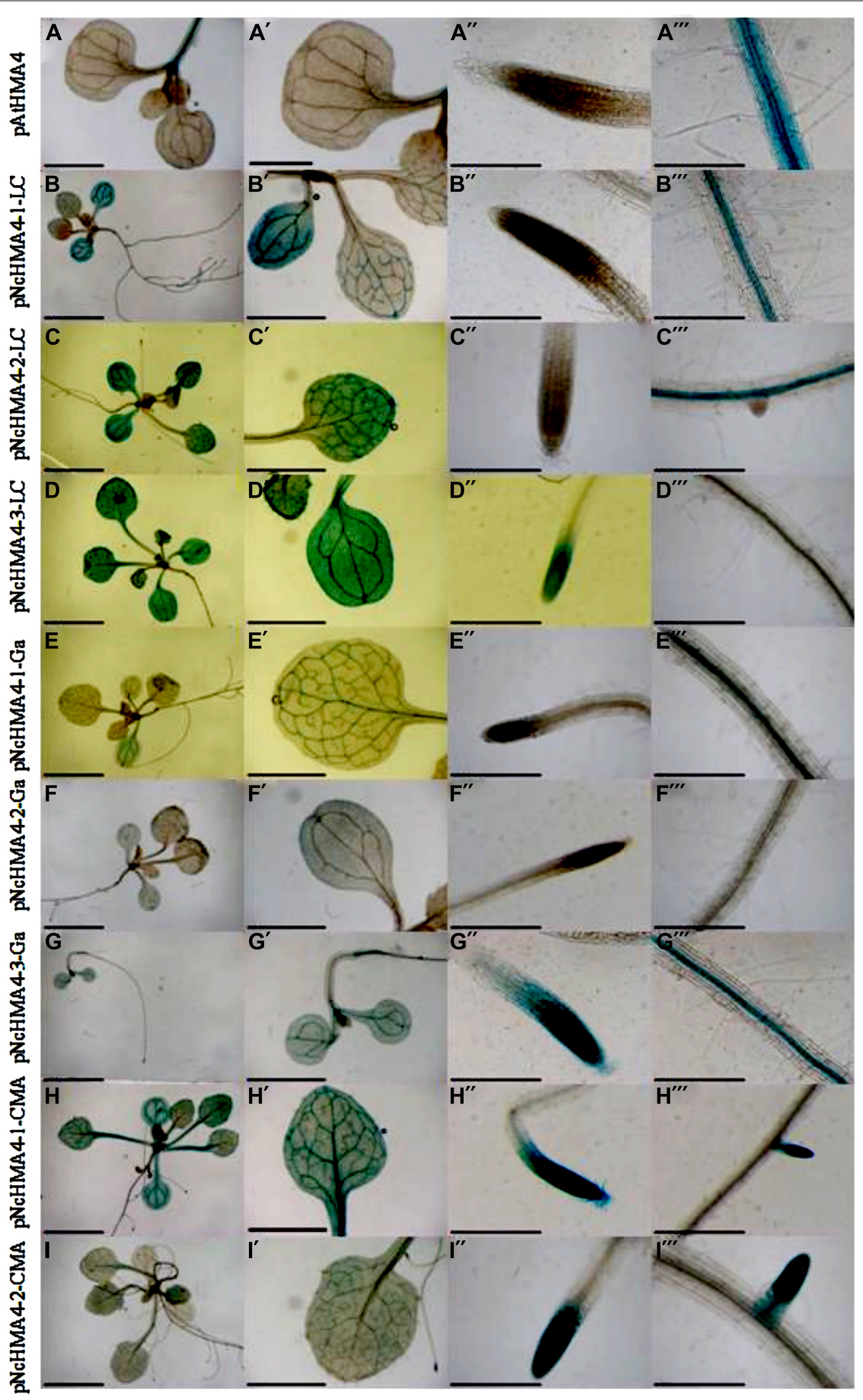

FIGURE 5 | Arabidopsis (wt) plants expressing GUS under AtHMA4 and NcHMA4 promoters from the accessions LC, Ga and CMA. [Scale bar $=5 \mathrm{~mm}$ in $(\mathbf{A})$ to $(\mathbf{I}), 2 \mathrm{~mm}$ in $\left(\mathbf{A}^{\prime}\right)$ to $\left(\mathbf{I}^{\prime}\right)$ and $30 \mu \mathrm{m}$ in $\left(\mathbf{A}^{\prime \prime}\right)$ to $\left(\mathbf{I}^{\prime \prime \prime}\right)$ ]. 


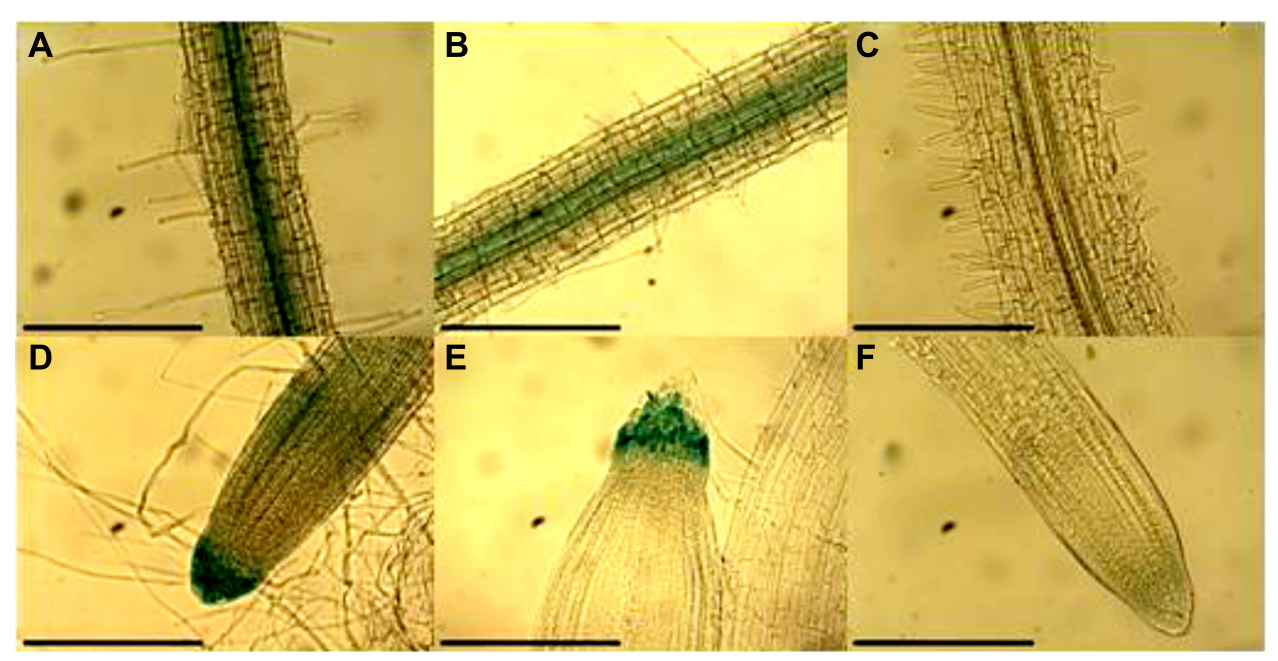

FIGURE 6 | GUS expression under the (A) and (D) pNcHMA4-1-LC, (B) and (E) pNcHMA4-2-LC in N. caerulescens roots. (C) and (F) are control roots [Scale bar $=30 \mu \mathrm{m}]$

A

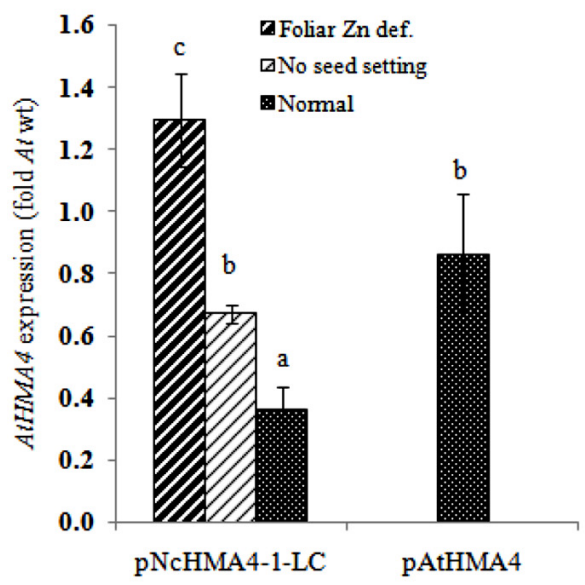

B

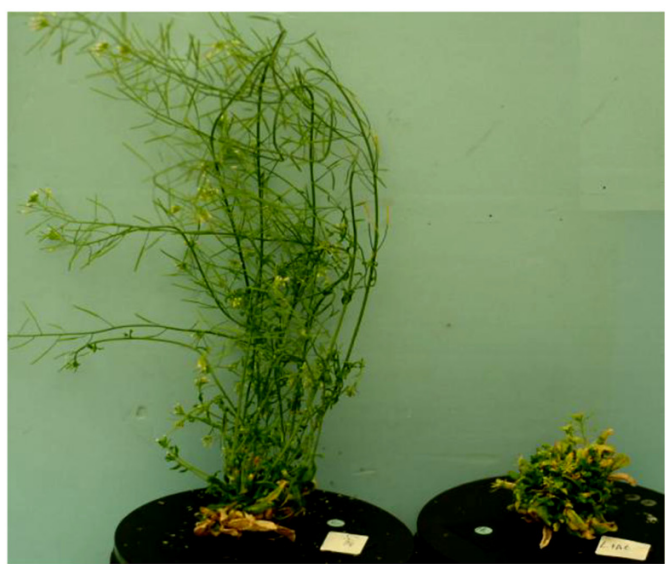

FIGURE 7 | (A) HMA4 expression in the root of transgenic Arabidopsis hma2hma4 double mutant plants (fold expression relative to At wild-type). (B) Arabidopsis hma2hma4 double mutant transformed by pNcHMA4-1-LC::AtHMA4, on the left a plant expressing AtHMA4 at a low level and on the right one with high AtHMA4 expression.

aggravates, rather than alleviates the foliar Zn deficiency of the double mutant (Figure 9).

\section{Cd TOLERANCE AND ACCUMULATION}

Cd tolerance, estimated from the root growth response, was tested in the same set of $\mathrm{T}_{1}$ lines that was used in the second $\mathrm{Zn}$ translocation experiment. Although there was significant variation in root length increments among the lines, the lines by Cd interaction was insignificant, showing that the lines did not significantly differ in their response to $\mathrm{Cd}$ (Figure 10). The Cd concentrations in shoot and root at the 0.5- and 12- $\mu \mathrm{M} \mathrm{Cd}$ exposure levels are given in Figures 11A,B. At the $0.5-\mu \mathrm{M}$ exposure level the lines transformed with the pAtHMA4 exhibited wild-type-like or higher foliar Cd concentrations, whereas the lines transformed with pNcHMA4-
1-LC exhibited significantly lower concentrations, in comparison with wild-type. In the roots the $\mathrm{Cd}$ concentrations were higher in wild-type than in the pNcHMA4-1-LC::NcHMA4-1-LC and pNcHMA4-1-LC::NcHMA4-2-LC lines. Overall, the patterns of variation in the $\mathrm{Cd}$ concentrations found at the $0.5-\mu \mathrm{M}$ exposure level were essentially the same as found for $\mathrm{Zn}$ among the same set of lines (see above). At the 12- $\mu \mathrm{M}$ exposure level, however, there were no considerable differences in the root and shoot Cd concentrations between the lines and wild-type, or among the lines (Figures 11A,B).

\section{DISCUSSION}

Our results clearly confirm that HMA4 is strongly over-expressed, both in roots and in shoots, in the $\mathrm{Zn} / \mathrm{Cd} / \mathrm{Ni}$ hyperaccumulator 


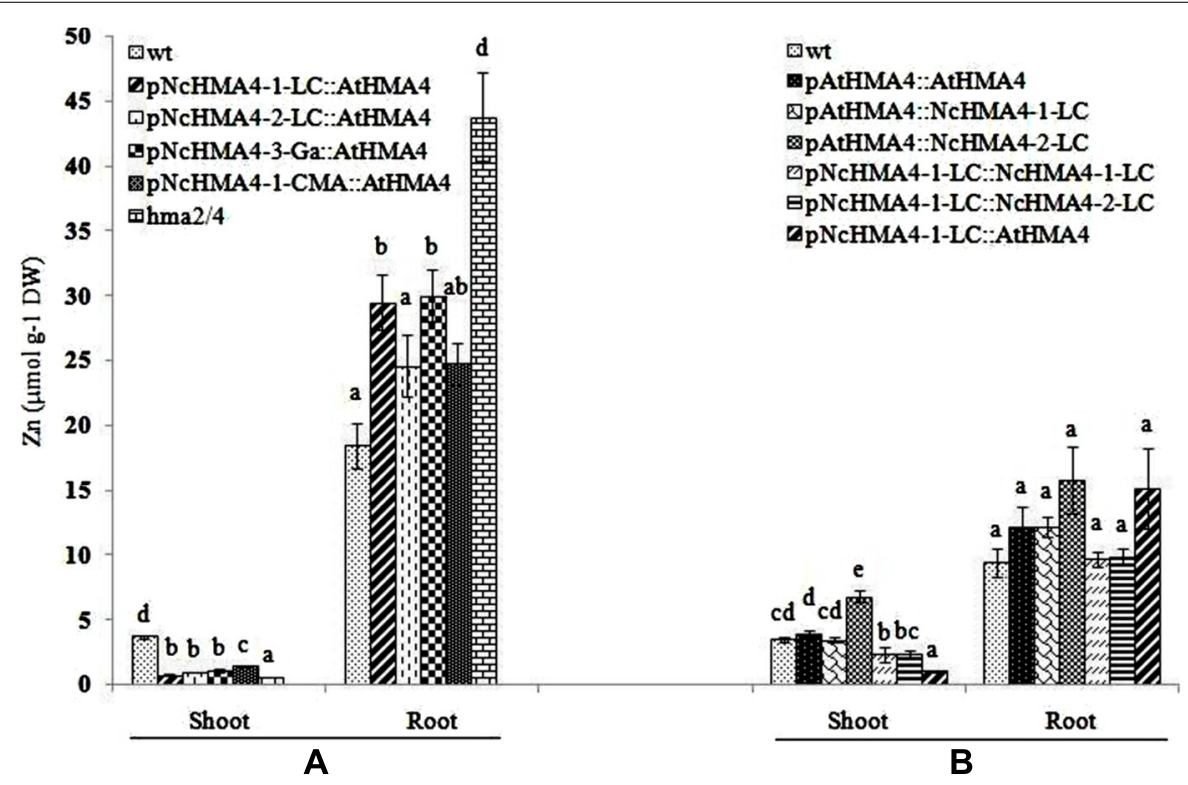

FIGURE 8 | Shoot $\mathrm{Zn}$ concentration ( $\mu \mathrm{mol} \mathrm{g}^{-1} \mathrm{DW}$ ) at $2 \mu \mathrm{M} \mathrm{ZnSO}_{4}$ in the nutrient solution in two successive experiments performed with Arabidopsis wt, untransformed hma2hma4 double mutant, and five transgenic lines expressing AtHMA4 under different $\mathrm{NcHMA4}$ promoters
(A), or six lines expressing three different HMA4 cDNAs, each under the AtHMA4 and the NcHMA4-1-LC promoter (B). Significant differences $(P<0.05)$ between means are indicated, separately for both shoots and roots, by different superscripted letters.

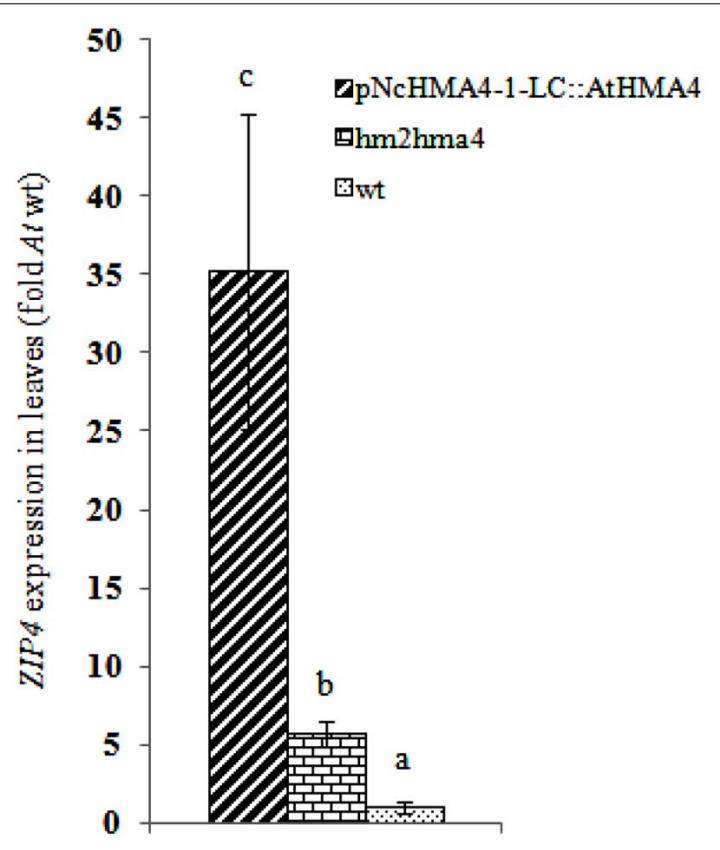

FIGURE 9 | Average ZIP4 expression in leaves in transgenic lines expressing AtHMA4 under the pNcHMA4-1-LC, the hma2hma4 double mutant, and wild-type (fold expression relative to Arabidopsis wt). Significant differences $(P<0.05)$ between means are indicated by different superscripted letters.

$N$. caerulescens, in comparison with the non-hyperaccumulator $A$. thaliana. Like in the other $\mathrm{Zn} / \mathrm{Cd}$ hyperaccumulator model, $A$. halleri, this over-expression must be due to a combination of copy

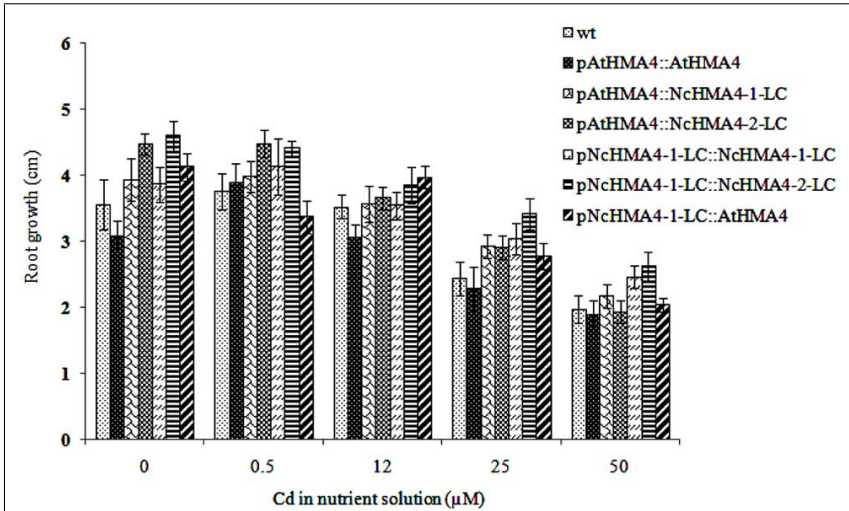

FIGURE 10 | Effect of Cd on root growth in Arabidopsis wt and transgenic lines. Root length increments were measured after 4 days.

number expansion and altered cis-regulation of the individual copies (Hanikenne et al., 2008). The copy number seems to vary between accessions in $N$. caerulescens, ranging from 2 to 4 (Ó Lochlainn etal., 2011; Craciun etal., 2012). The three copies that we found in the LC library matches with the three copies established in the nearby accession from Prayon (Craciun et al., 2012).

The most striking result that we obtained is that expressing either AtHMA4 or NcHMA4 cDNAs under any N. caerulescens HMA4 promoter did not fully complement, except for two $\mathrm{T}_{1}$ plants, the Zn-translocation-deficient $A$. thaliana hma2hma4 double mutant, whereas expression under the endogenous AtHMA4 promoter always did. This is not simply a matter of the degree 


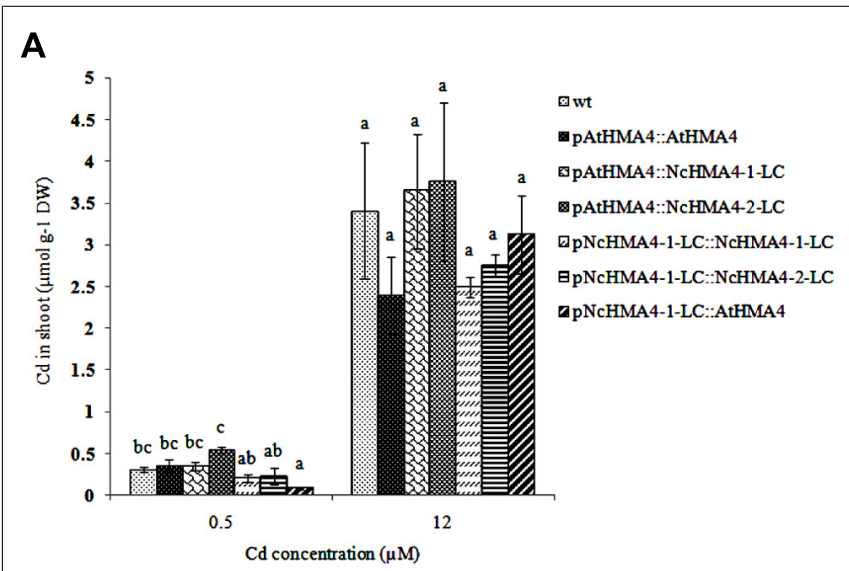

B

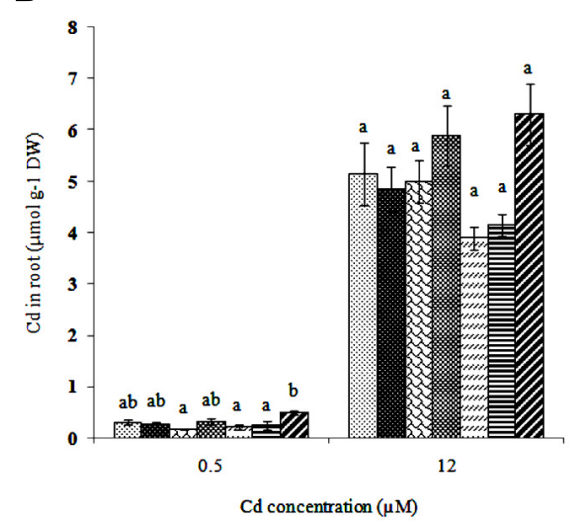

FIGURE 11 | Cd concentration in Arabidopsis wt and transgenic lines ( $\mu \mathrm{mol} \mathrm{g}^{-1} \mathrm{DW}$ ) exposed to $\mathbf{0 . 5}$ and $12 \mu \mathbf{M} \mathbf{C d}$. (A) shoot, (B) root. Significant differences $(P<0.05)$ between means are indicated, separately for both treatments, by different superscripted letters.

of expression of the transgene in the root. Admittedly, the HMA4 expression levels produced by $\mathrm{Nc}$ promoters in the double mutant background were surprisingly low, in some cases even significantly lower than the wild-type expression level in A. thaliana. However, when compared in lines derived from plants with equal transgene expression levels in the roots, pAtHMA4::HMA4 constructs completely restored the $\mathrm{Zn}$ root-to-shoot translocation to wild-type level or higher, whereas pNcHMA4::HMA4 constructs only slightly enhanced the foliar $\mathrm{Zn}$ concentration. Even in pNcHMA4::HMA4 lines expressing the transgene about 10 times higher than wild-type in the root, the foliar $\mathrm{Zn}$ concentrations were not higher than $60 \%$ of the wild-type level, whereas a strongly expressing pAtHMA4::HMA4 line showed enhanced Zn translocation, in comparison with wild-type (Figure 8B). Admittedly, there was considerable variation regarding the root and shoot $\mathrm{Zn}$ concentrations between the two successive experiments that we performed (Figure 8). We cannot explain this variation, however, it is remarkable that the $\mathrm{Zn}$ shoot concentrations and translocation rates of the lines with the $N c$ promoters are consistently intermediate between those in wild-type and in the hma2hma4 double mutant. The reasons for this are not entirely clear, but ectopic expression under the $\mathrm{Nc}$ promoters, in terms of tissue-specificity, seems to be the most plausible explanation. As indicated by the GUS assays, the Nc promoters are often mainly active in all the cells of the 2-mm apical root segment, rather than in the xylem parenchyma, whereas the At promoter is exclusively active in the xylem parenchyma (Figure 5), as is the Nc promoter tested in the $A$. rhizogenes-transformed $N$. caerulescens roots. One might argue that this could be owing to the short length of the $\mathrm{Nc}$ promoter sequences that we isolated, i.e., $2100-2600 \mathrm{bp}$, in comparison with the At promoter (4103 bp), which covered the whole upstream intergenic region. However, as shown by the GUS assay with Rhizogenes-transformed $N$. caerulescens roots, the $2135 \mathrm{bp}$ of the $p N c H M A 4-1-L C$ does produce expression predominantly in the xylem parenchyma in N. caerulescens itself (Figure 6), suggesting that $A$. thaliana and N. caerulescens may have different transcriptional regulators for HMA4 expression, at least in part.

There seemed to be a degree of positive correlation between the transgene expression levels in the root and the foliar $\mathrm{Zn}$ concentrations among the lines transformed with the Nc promoters (Figure 8A). However, in spite of this, the transgene expression levels in the roots were also positively correlated with the severity of foliar $\mathrm{Zn}$ deficiency symptoms, at least among the plants transformed with the pNcHMA4-1-LC::AtHMA4 construct (Figure 7). The most likely explanation for this phenomenon is that the transgene expression levels in roots are more or less correlated with those in shoots (Figures 3 and 4). It is remarkable that expression of AtHMA4 under the pNcHMA4-1-LC improved, albeit slightly, the root-to-shoot $\mathrm{Zn}$ translocation (Figure 8B), but aggravated rather than alleviated the foliar Zn deficiency symptoms in the hma2hma4 double mutant, as demonstrated by the strongly enhanced ZIP4 expression level (Figure 9). The latter might be attributable to the relatively high level of HMA4 expression in the leaf mesophyll (Figure 4), which would be expected to enhance the efflux of $\mathrm{Zn}$ from photosynthetically active cells. This might in turn cause or enhance $\mathrm{Zn}$ deficiency in the absence of hyperaccumulator-like uptake and root-to-shoot translocation rates. The high expression of HMA4 outside the veins in the leaves of mutants transformed with $p N c H M A 4:: H M A 4$ constructs might well reflect the natural expression pattern in $N$. caerulescens itself. As demonstrated by Klein et al. (2008), N. caerulescens cells in suspension culture accumulate less $\mathrm{Zn}$ or $\mathrm{Cd}$ than A. thaliana cells, presumably due to higher metal efflux rates. It is therefore conceivable that a high-level of HMA4 expression in the leaf mesophyll in hyperaccumulators could serve to promote the cell-to-cell transport of metals from the vasculature to their final destiny, the large epidermal cells (Küpper and Kochian, 2010).

In the Cd tolerance test, we used only wild-type as a control, since we had insufficient seeds of the hma2hma4 double mutant. However, it can be safely assumed that the double mutant, at least when in the wild-type background, does not have a detectable phenotype for Cd tolerance (Hussain et al., 2004; Wong and Cobbett, 2009). In any case, our results clearly show that HMA4 expression, regardless of the promoter and cDNA source, does not confer Cd tolerance, in comparison to wild-type, to the hma2hma4 double mutant. This is not surprising, because at the higher toxic Cd exposure levels there were no considerable effects of the transgenes on the root or shoot $\mathrm{Cd}$ concentrations (Figure 11). At the non-toxic 
$0.5-\mu \mathrm{M} C d$ exposure level, however, there were pronounced and significant effects of the transgenes on the root and shoot $\mathrm{Cd}$ concentrations. Overall, these effects were about the same as those on the root and shoot $\mathrm{Zn}$ concentrations (Figure 8). This, together with the extremely low foliar $\mathrm{Cd}$ concentration in the mutant at the $0.5 \mu \mathrm{M}$ exposure level, confirms that HMA4 can translocate both $\mathrm{Zn}$ and $\mathrm{Cd}$ in A. thaliana (Hussain et al., 2004; Wong and Cobbett, 2009). However, at higher exposure levels, the translocation of $\mathrm{Cd}$, in contrast with that of $\mathrm{Zn}$, is apparently no longer dependent on HMA4, but mediated by other systems with lower affinity.

Comparing our results with those obtained with HMA4 genes and promoters from the other model $\mathrm{Zn} / \mathrm{Cd}$ hyperaccumulator, A. halleri, reveals several differences. First, the AhHMA4 promoters produced a much higher GUS expression in A. thaliana roots than did the NcHMA4 promoters in the present study. Second, GUS expression in transgenic $A$. thaliana under the different AhHMA4 promoters exhibited the correct tissue-specificity pattern, i.e., exclusively in the xylem parenchyma and the root cap (Hanikenne et al., 2008). The reason for the low and different HMA4 expression under NcHMA4 promoters that we observed in our study is elusive, but it is well conceivable that it has something to do with the bigger phylogenetic distance between $N$. caerulescens and $A$. thaliana, in comparison with that between the congeneric A. thaliana and A. halleri.

Finally, the question of whether correct over-expression of HMA4 can confer hyperaccumulator-like metal translocation rates to non-hyperaccumulator host species remains unanswered. HMA4 over-expression in A. thaliana has been reported to enhance $\mathrm{Cd}$ or $\mathrm{Zn}$ translocation to the shoot, albeit only to a marginal

\section{REFERENCES}

Andrés-Colás, N., Sancenon, V., Rodriguez-Navarro, S., Mayo, S., Thiele, D. J., Ecker, J. R., et al. (2006). The Arabidopsis heavy metal P-type ATPase HMA5 interacts with metallochaperones and functions in copper detoxification of roots. Plant J. 45, 225-236. doi: 10.1111/j. 1365-313X.2005.02601.x

Antonovics, J., Bradshaw, A. D., and Turner, R. G. (1971). "Heavy metal tolerance in plants," in Advances in Ecological Research, ed. J. B. Cragg (New York: Academic Press), 1-85.

Assunção, A. G. L., Bookum, W. M., Nelissen, H. J. M., Vooijs, R., Schat, H., and Ernst, W. H. O. (2003). Differential metal-specific tolerance and accumulation patterns among Thlaspi caerulescens populations originating from different soil types. New Phytol. 159, 411-419. doi: 10.1046/j.1469-8137.2003.00819.x

Assunção, A. G. L., Herrero, E., Lin, Y.-F., Huettel, B., Talukdar, S., Smaczniak, C., et al. (2010). Arabidopsis thaliana transcription factors bZIP19 and bZIP23 regulate the adaptation to zinc deficiency. Proc. Natl. Acad. Sci. 107,
10296-10301. doi: 10.1073/pnas. 1004788107

Baker, A. J. M., McGrath, S. P., Reeves, D. R., and Smith, J. A. C. (2000). "Metal hyperaccumulator plants: a review of the ecology and physiology of a biological resource for phytoremediation of metal-polluted soils," in Phytoremediation of Contaminated Soils and Water, eds N. Terry and G. Banuelos (Boca Raton, FL: CRC Press LLC), 171-188.

Barabasz, A., Wilkowska, A. Hanikenne, M., Czarny, M., et al. (2012). Metal response of transgenic tomato plantsexpressing P1B-ATPase. Physiol. Plant. 145, 315331. doi: 10.1111/j.1399-3054.2012. 01584.x

Bernard, C., Roosens, N., Czernic, P., Lebrun, M., and Verbruggen, N. (2004). A novel CPx-ATPase from the cadmium hyperaccumulator Thlaspi caerulescens. FEBS Lett. 569, 140 148. doi: 10.1016/j.febslet.2004. 05.036

Clemens, S. (2001). Molecular mechanisms of plant metal tolerance and homeostasis. Planta 212, 475-486. doi: $10.1007 /$ s004250000458 Ruszczyñska, A., Bulska, E.

degree (Verret et al., 2004; Hanikenne et al., 2008). However, in the latter studies HMA4 has been expressed under the 35S CaMV promoter, which is bound to yield ectopic expression. Barabasz et al. (2012) expressed a HMA4 copy from A. halleri under the endogenous $A h$ promoter in tobacco, but also found no more than a marginal effect on $\mathrm{Zn}$ translocation. It is unknown, however, whether the transgene was correctly over-expressed in the latter study. Unfortunately, the NcHMA4 promoters that we used in the present study did not produce the correct expression pattern in A. thaliana roots, which probably explains the inconsiderable effects of the HMA4 transgenes on $\mathrm{Zn}$ and $\mathrm{Cd}$ translocation. It is reasonable to expect that this may not apply to the A. halleri HMA4 promoters (see above). However, even in cases where hyperaccumulator promoters would yield a correct hyperaccumulator-like HMA4 expression pattern, it is doubtful whether this would lead to more than marginal increments of root-to-shoot metal translocation rates. There are good reasons to assume that hyperaccumulator-like translocation rates are not only dependent on HMA4-mediated xylem loading, but also on "upstream mechanisms," which prevent the retention of metals in root cell vacuoles (Richau et al., 2009; Deinlein et al., 2012).

\section{ACKNOWLEDGMENTS}

We thank the "Higher Education Commission (HEC), Pakistan" for the financial support of the first author.

\section{SUPPLEMENTARY MATERIAL}

The Supplementary Material for this article can be found online at http://www.frontiersin.org/Journal/10.3389/fpls.2013. 00404/abstract

Clemens, S. (2006). Toxic metal accumulation, responses to exposure and mechanisms of tolerance in plants. Biochimie 88, 1707-1719. doi: 10.1016/j.biochi.2006.07.003

Clough, S. J., and Bent, A. F. (1998). Floral dip: a simplified method for Agrobacterium-mediated transformation of Arabidopsis thaliana. Plant J. 16, 735-743. doi: 10.1046/ j.1365-313x.1998.00343.x

Cosio, C., Martinoia, E., and Keller, C. (2004). Hyperaccumulation of cadmium and zinc in Thlaspi caerulescens and Arabidopsis halleri at the leaf cellular level. Plant Physiol. 134, 716-725. doi: 10.1104/pp.103. 031948

Courbot, M., Willems, G., Motte, P., Arvidsson, S., Roosens, N. Saumitou-Laprade, P., et al. (2007) A major quantitative trait locus for cadmium tolerance in Arabidopsis halleri colocalizes with HMA4, a gene encoding a heavy metal ATPase. Plant Physiol. 144, 1052-1065. doi: 10.1104/pp.106.095133

Craciun, A. R., Meyer, C. L., Chen, J. Roosens, N., De Groodt, R., Hilson, P., et al. (2012). Variation in HMA4 gene copy number and expression among Noccaea caerulescens populations presenting different levels of Cd tolerance and accumulation. J. Exp. Bot. 63, 4179-4189. doi: 10.1093/jxb/ers104

Deinlein, U., Weber, M., Schmidt, H., Rensch, S., Trampczynska, A., Hansen, T. H., et al. (2012). Elevated nicotianamine levels in Arabidopsis halleri roots play a key role in zinc hyperaccumulation. Plant Cell 24, 708-723. doi: 10.1105/tpc. 111.095000

Ernst, W. H. O. (1974). Schwermetallvegetation der Erde. Stuttgart, Germany.

Guerinot, M. L. (2000). The ZIP family of metal transporters. Biochim. Biophys. Acta 1465, 190-198. doi: 10.1016/S0005-2736(00)00138-3

Gustin, J. L., Loureiro, M. E., Kim, D., Na, G., Tikhonova, M., and Salt, D. E. (2009). MTP1-dependent Zn sequestration into shoot vacuoles suggests dual roles in $\mathrm{Zn}$ tolerance and accumulation in Zn-hyperaccumulating plants. Plant J. 57, 1116-1127. doi: 10.1111/j.1365-313X.2008.03754.X

Hanikenne, M., Talke, I. N., Haydon, M. J., Lanz, C., Nolte, A., Motte, P., et al. (2008). Evolution 
of metal hyperaccumulation required cis-regulatory changes and triplication of HMA4. Nature 453, 391-395. doi: 10.1038/nature06877

Hussain, D., Haydon, M. J., Wang, Y., Wong, E., Sherson, S. M., Young, J., etal. (2004). P-type ATPase heavy metal transporters with roles in essential zinc homeostasis in Arabidopsis. Plant Cell 16, 1327-1339. doi: $10.1105 /$ tpc.020487

Jack, E., Hakvoort, H. W. J., Reumer, A., Verkleij, J. a. C., Schat, H., and Ernst, W. H. O. (2007). Real-time PCR analysis of metallothionein-2b expression in metallicolous and nonmetallicolous populations of Silene vulgaris (Moench) Garcke. Environ. Exp. Bot. 59, 84-91. doi: 10.1016/j.envexpbot.2005.10.005

Kamal, M., Ghaly, A. E., Mahmoud, N., and Côté, R. (2004). Phytoaccumulation of heavy metals by aquatic plants. Environ. Int. 29, 1029-1039. doi: 10.1016/S0160-4120(03)00091-6

Karimi, M., Inze, D., and Depicker, A. (2002). GATEWAY vectors for Agrobacterium-mediated plant transformation. Trends Plant Sci. 7, 193-195. doi: 10.1016/S13601385(02)02251-3

Kim, Y. Y., Choi, H., Segami, S., Cho, H. T., Martinoia, E., Maeshima, M. et al. (2009). AtHMAl contributes to the detoxification of excess $\mathrm{Zn}$ (II) in Arabidopsis. Plant J. 58, 737-753. doi: 10.1111/j.1365-313X.2009.03818.x

Klein, M. A., Sekimoto, H., Milner, M. J., and Kochian, L. V. (2008) Investigation of heavy metal hyperaccumulation at the cellular level: development and characterization of Thlaspi caerulescens suspension cell lines. Plant Physiol. 147, 2006-2016. doi: 10.1104/pp.108.119719

Küpper, H., and Kochian, L. V. (2010). Transcriptional regulation of metal transport genes and mineral nutrition during acclimatization to cadmium and zinc in the $\mathrm{Cd} / \mathrm{Zn}$ hyperaccumulator, Thlaspi caerulescens (Ganges population). New Phytol. 185, 114-129. doi: 10.1111/j.1469-8137.2009.03051.x

Kumar, S., Nei, M., Dudley, J., and Tamura, K. (2008). MEGA: a biologist-centric software for evolutionary analysis of DNA and protein sequences. Brief Bioinform. 9, 299-306. doi: 10.1093/bib/bbn017

Limpens, E., Ramos, J., Franken, C., Raz, V., Compaan, B., Franssen, H., et al.
(2004). RNA interference in Agrobacterium rhizogenes-transformed roots of Arabidopsis and Medicago truncatula. J. Exp. Bot. 55, 983-992. doi: 10.1093/jxb/erh122

Livak, K. J., and Schmittgen, T. D. (2001). Analysis of relative gene expression data using real-time quantitative PCR and the 2(-Delta Delta $\mathrm{C}(\mathrm{T})$ ) method. Methods 25, 402-408 doi: 10.1006/meth.2001.1262

Macnair, M. R. (1993). The genetics of metal tolerance in vascular plants. New Phytol. 124, 541-559. doi: 10.1111/j.1469-8137.1993.tb03846.x Marschner, H. (1995). Mineral Nutri tion of Higher Plants, 2nd Edn. Academic Press, London.

Mohtadi, A., Ghaderian, S., and Schat, H. (2012). A comparison of lead accumulation and tolerance among heavy metal hyperaccumulating and non-hyperaccumulating metallophytes. Plant Soil 352, 267-276. doi: 10.1007/s11104-0110994-5

Ó Lochlainn, S., Bowen, H. C., Fray, R. G., Hammond, J. P., King, G. J., White, P. J., et al. (2011). Tandem quadruplication of HMA4 in the zinc $(\mathrm{Zn})$ and cadmium (Cd) hyperaccumulator Noccaea caerulescens. PLoS ONE 6:e17814. doi: 10.1371/journal.pone.0017814

Papoyan, A., and Kochian, L. V. (2004). Identification of Thlaspi caerulescens genes that may be involved in heavy metal hyperaccumulation and tolerance. Characterization of a novel heavy metal transporting ATPase. Plant Physiol. 136, 3814-3823. doi: 10.1104/pp.104.044503

Peer, W. A., Mamoudian, M., Lahner, B., Reeves, R. D., Murphy, A S., and Salt, D. E. (2003). Identifying model metal hyperaccumulating plants: germplasm analysis of 20 Brassicaceae accessions from a wide geographical area. New Phytol. 159, 421-430. doi: 10.1046/j.14698137.2003.00822.x

Richau, K. H., Kozhevnikova, A D., Seregin, I. V., Vooijs, R., Koevoets, P. L., Smith, J. A., et al. (2009). Chelation by histidine inhibits the vacuolar sequestration of nickel in roots of the hyperaccumulator Thlaspi caerulescens. New Phytol. 183, 106-116. doi: 10.1111/j.14698137.2009.02826.x

Rivera, R., Edwards, K. J., Barker, J. H., Arnold, G. M., Ayad, G., Hodgkin, T., et al. (1999). Isolation and characterization of polymorphic microsatellites in Cocos nucifera L. Genome 42, 668-675. doi: 10.1139/g98-170

Sambrook, J., Fritsch, E. F., and Maniatis, T. (1989). Molecular Cloning: A Laboratory Manual, 2nd Edn. New York: Cold Spring Harbor Laboratory Press.

Schat, H., and Ten Bookum, W. M. T. (1992). Genetic control of copper tolerance in Silene vulgaris. Heredity 68 219-229. doi: 10.1038/hdy.1992.35

Schat, H., and Vooijs, R. (1997). Multiple tolerance and co-tolerance to heavy metals in Silene vulgaris: a co-segregation analysis. New Phytol. 136, 489-496. doi: 10.1046/j.14698137.1997.00756.x

Schat, H., Vooijs, R., and Kuiper, E. (1996). Identical major gene loci for heavy metal tolerances that have independently evolved in different local populations and subspecies of Silene vulgaris. Evolution 50, 1888 1895. doi: $10.2307 / 2410747$

Shahzad, Z., Gosti, F., Frerot, H. Lacombe, E., Roosens, N., SaumitouLaprade, P., et al. (2010). The five AhMTP1 zinc transporter undergo different evolutionary fates towards adaptive evolution to zinc tolerance in Arabidopsis hal leri. PLoS Genet. 6:e1000911. doi: 10.1371/journal.pgen.1000911

Sokal, R. R., and Rohlf, F. J. (1981). Biometry, 2nd Edn. San Francisco, Freeman.

Ueno, D., Milner, M. J., Yamaji, N. Yokosho, K., Koyama, E., Clemencia Zambrano, M., etal. (2011). Elevated expression of TcHMA3 plays a key role in the extreme $\mathrm{Cd}$ tolerance in a Cd-hyperaccumulating ecotype of Thlaspi caerulescens. Plant J. 66, 852-862. doi: 10.1111/j.1365313X.2011.04548.x

van de Mortel, J. E., Almar Villanueva L., Schat, H., Kwekkeboom, J., Coughlan, S., Moerland, P. D., et al. (2006). Large expression differences in genes for iron and zinc homeostasis, stress response, and lignin biosynthesis distinguish roots of Arabidopsis thaliana and the related metal hyperaccumulator Thlaspi caerulescens. Plant Physiol. 142, 1127-1147. doi: 10.1104/pp.106.082073

van der Ent, A., Baker, A. M., Reeves, R., Pollard, A. J., and Schat, H. (2013). Hyperaccumulators of metal and metalloid trace elements: facts and fiction. Plant Soil 362, 319-334. doi: 10.1007/s11104-012-1287-3

Verret, F., Gravot, A., Auroy, P., Leonhardt, N., David, P., Nussaume, L., et al. (2004). Overexpression of AtHMA4 enhances rootto-shoot translocation of zinc and cadmium and plant metal tolerance. FEBS Lett. 576, 306-312. doi: 10.1016/j.febslet.2004.09.023

Willems, G., Dräger, D. B., Courbot, M., Godé, C., Verbruggen, N., and Saumitou-Laprade, P. (2007). The genetic basis of zinc tolerance in the metallophyte Arabidopsis halleri ssp. halleri (Brassicaceae): an analysis of quantitative trait loci. Genetics 176, 659-674. doi: 10.1534/genetics. 106.064485

Wong, C. K. E., and Cobbett, C. S. (2009). HMA P-type ATPases are the major mechanism for root-toshoot Cd translocation in Arabidopsis thaliana. New Phytol. 181, 71-78. doi: 10.1111/j.1469-8137.2008.02638.x

Conflict of Interest Statement: The authors declare that the research was conducted in the absence of any commercial or financial relationships that could be construed as a potential conflict of interest.

Received: 30 April 2013; accepted: 24 September 2013; published online: 16 October 2013.

Citation: Iqbal M, Nawaz I, Hassan Z, Hakvoort HWJ, Bliek M, Aarts MGM and Schat H (2013) Expression of HMA4 cDNAs of the zinc hyperaccumulator Noccaea caerulescens from endogenous NcHMA4 promoters does not complement the zinc-deficiency phenotype of the Arabidopsis thaliana hma2hma4 double mutant. Front. Plant Sci. 4:404. doi: 10.3389/fpls.2013.00404

This article was submitted to Plant Physiology, a section of the journal Frontiers in Plant Science.

Copyright (c) 2013 Iqbal, Nawaz, Hassan, Hakvoort, Bliek, Aarts and Schat. This is an open-access article distributed under the terms of the Creative Commons Attribution License (CC BY). The use, distribution or reproduction in other forums is permitted, provided the original author(s) or licensor are credited and that the original publication in this journal is cited, in accordance with accepted academic practice. No use, distribution or reproduction is permitted which does not comply with these terms. 